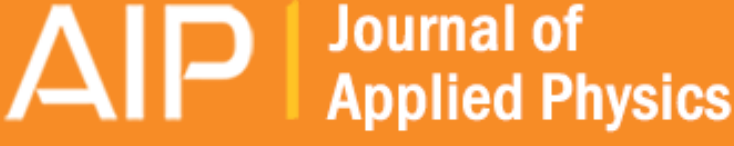

\section{Lattice thermal conductivity of a silicon nanowire under surface stress}

Monrudee Liangruksa and Ishwar K. Puri

Citation: Journal of Applied Physics 109, 113501 (2011); doi: 10.1063/1.3583668

View online: http://dx.doi.org/10.1063/1.3583668

View Table of Contents: http://scitation.aip.org/content/aip/journal/jap/109/11?ver=pdfcov

Published by the AIP Publishing

\section{Articles you may be interested in}

Thermal conductivity of semiconductor nanowires from micro to nano length scales

J. Appl. Phys. 111, 024311 (2012); 10.1063/1.3677973

Full dispersion versus Debye model evaluation of lattice thermal conductivity with a Landauer approach

J. Appl. Phys. 109, 073718 (2011); 10.1063/1.3567111

Lattice thermal conductivity of freestanding gallium nitride nanowires

J. Appl. Phys. 108, 034324 (2010); 10.1063/1.3463358

Lattice thermal conductivity in a silicon nanowire with square cross section

J. Appl. Phys. 100, 014305 (2006); 10.1063/1.2211648

The lattice thermal conductivity of a semiconductor nanowire

J. Appl. Phys. 99, 114318 (2006); 10.1063/1.2203721

MIT LINCOLN

LABORATORY CAREERS

Discover the satisfaction of innovation and service

to the nation
- Space Control

- Air \& Missile Defense

- Communications Systems \& Cyber Security

- Intelligence, Surveillance and

Reconnaissance Systems

- Advanced
Electronics
- Tactical Systems
- Homeland
Protection
- Air Traffic Control

LINCOLN LABORATORY

MassachusetTs Institute of TeChNOLOgY

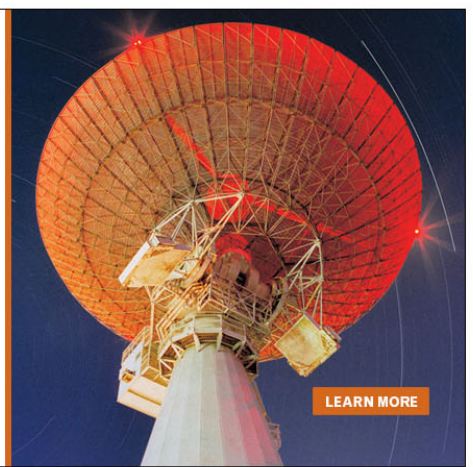




\title{
Lattice thermal conductivity of a silicon nanowire under surface stress
}

\author{
Monrudee Liangruksa and Ishwar K. Puria) \\ Department of Engineering Science and Mechanics, Virginia Polytechnic Institute and State University, \\ Blacksburg, Virginia 24061, USA
}

(Received 16 February 2011; accepted 29 March 2011; published online 1 June 2011)

\begin{abstract}
The effects of surface stress on the lattice thermal conductivity are investigated for a silicon nanowire. A phonon dispersion relation is derived based on a continuum approach for a nanowire under surface stress. The phonon Boltzmann equation and the relaxation time are employed to calculate the lattice thermal conductivity. Surface stress, which has a significant influence on the phonon dispersion and thus the Debye temperature, decreases the lattice thermal conductivity. The conductivity varies with changing surface stress, e.g., due to adsorption layers and material coatings. This suggests a phonon engineering approach to tune the conductivity of nanomaterials.

(C) 2011 American Institute of Physics. [doi:10.1063/1.3583668]
\end{abstract}

\section{INTRODUCTION}

Low-dimensional systems, such as silicon ( $\mathrm{Si}$ ) nanowires, display unique mechanical, electrical and optical properties that are not displayed by their bulk counterparts. ${ }^{1,2}$ The smaller feature sizes of devices based on these nanostructures also lead to thermal management issues, e.g., due to the increase in the power dissipation per unit surface area, or the need to reduce the phonon thermal conductivity in thermoelectric devices. Thus, strategies to tune the thermal conductivity of nanomaterials can help optimize the performance and reliability of nanoscale devices. ${ }^{3}$

The thermal conductivity can be decreased by doping a material (i.e., by substituting atoms of another element in its lattice) or by increasing the phonon scattering. ${ }^{4}$ Phonon engineering approaches, such as those that control and manipulate the phonon group velocity, and the polarization and density of states, also influence the thermal properties of a device. ${ }^{5}$ These approaches can involve the spatial confinement and boundary scattering of phonons in quantum wells, ${ }^{6}$ and variations in the cross sectional geometry of nanowires. $^{3,7}$ The phonon properties of a nanostructure, such as a nanowire, ${ }^{8}$ can be tailored through a proper selection of acoustically mismatched materials. ${ }^{9-13}$ The surface roughness of a nanowire can also significantly influence its thermal conductivity. ${ }^{14,15}$ For instance, the room temperature phonon thermal conductivity has been reported to be reduced for roughened silicon nanowires to $1.6 \mathrm{~W} \mathrm{~m}^{-1} \mathrm{~K}^{-1}$ from the corresponding bulk value of $150 \mathrm{~W} \mathrm{~m}^{-1} \mathrm{~K}^{-1}$ (Ref. 14).

When mechanical stress is varied in confined nanostructures, this alters the velocity of acoustic waves. ${ }^{16}$ Hence, it should be possible to also employ such a variation for phonon engineering. The acoustoelastic effect that the stress produces is negligible in bulk materials where its values are typically below $\approx 100 \mathrm{MPa}$. These account for only a $0.1 \%$ change in the phase velocity of acoustic waves. ${ }^{17}$ A considerably larger stress, e.g., as much as $1 \mathrm{GPa}$, can arise in

\footnotetext{
a) Author to whom correspondence should be addressed. Electronic mail: ikpuri@vt.edu.
}

layered nanostructures through surface intermolecular forces when there is a misfit between the film and substrate lattices. This larger stress can induce much more significant changes in the phonon phase velocity.

The different coordination of atoms on the surface of a material with those inside it leads to a redistributed charge density below the surface. ${ }^{18}$ As the charges rearrange in response to missing atoms, the resulting net forces on the surface atoms induce surface stress. The stress depends on the electronic structure of the material and the crystallographic orientation of its surface. Depending on the bond strengths between the surface and neighboring atoms, surface stress can be either tensile or compressive. Atoms on the topmost surface layer of a lattice are subject to tensile surface stress when they attract one another and tend to shrink the surface. ${ }^{18}$ In other words, surface stress in a thin film system can occur due to the adsorption of atoms or molecules on surfaces. ${ }^{19,20}$

Surface stress can be modeled as an external loading on the free end of a beam, ${ }^{21}$ or by distributing a load along it. $^{21,22}$ The influence of stress on the natural frequency of the beam, ${ }^{19,20,23,24}$ its stiffness, ${ }^{25}$ and deformation ${ }^{20}$ can, in turn, be used to tune other physical properties through secondary effects. Examples include the surface wave velocity, and electrical and thermal conductivities.

The literature does not discuss the influence of surface stress on phonon dispersion. Therefore, its consequences for a silicon nanowire, which is a promising nanoelectronic and thermoelectric material, ${ }^{26}$ are investigated. The phonon wavelength is assumed to be much longer than the lattice constant (which represents the characteristic distance between atoms) for acoustic phonons in the sub-THz frequency range. For such cases, the macroscopic theory of wave scattering from rough surfaces is in good agreement with the acoustic phonon-interface interaction method. ${ }^{27} \mathrm{We}$ employ this continuum approach to understand how modifications of acoustic phonon dispersion by the surface stress in nanostructures can alter the phonon thermal conductivity. Longitudinal and flexural wave propagations due to surface stress have also been previously investigated in nanofibers within a continuum framework. ${ }^{28,29}$ 


\section{METHODOLOGY}

\section{A. Phonon dispersion}

The equations of elastic vibration in an isotropic medium are

$$
\rho \ddot{u}_{i}=(\lambda+\mu) \tilde{\theta}_{, i}+\mu \nabla^{2} u_{i},
$$

where $\lambda$ denotes Lamé's first parameter, $\rho$ mass density, $\mu$ Lamé's second parameter, $\tilde{\theta}=\varepsilon_{i i}=u_{i, i}$, and $\nabla^{2}=\partial^{2} / \partial x_{1}^{2}$ $+\partial^{2} / \partial x_{2}^{2}+\partial^{2} / \partial x_{3}^{2}$. Differentiating Eq. (1) with respect to $x_{i}$ and summing over $i$ provides a wave equation for the dilatation $\tilde{\theta}$,

$$
\partial^{2} \tilde{\theta} / \partial t^{2}=c_{\alpha}^{2} \nabla^{2} \tilde{\theta}
$$

where $c_{\alpha}=\sqrt{(\lambda+2 \mu) / \rho}$ denotes the wave speed. For a structure undergoing rotation $w_{i}$ about its axis $x_{i}$, $w_{i}=-1 / 2 \varepsilon_{i j k} u_{k, j}$. In terms of the rotations $w_{i}$, Eq. (1) may be written in the form

$$
\partial^{2} w_{1} / \partial t^{2}=c_{\beta}^{2} \nabla^{2} w_{1},
$$

where $c_{\beta}=\sqrt{\mu / \rho}$. The velocity ratio $\chi_{0}=c_{\alpha} / c_{\beta}=$ $\sqrt{(\lambda+2 \mu) / \mu}=\sqrt{(2-2 v) /(1-2 v)}$, where the Poisson's ratio $v$ relates the two types of bulk waves in an isotropic elastic media, one associated with volumetric changes and the other with shear motion.

A wave traveling with a velocity $c_{\alpha}$ is a dilatational or primary wave ( $P$-wave) while that with a velocity $c_{\beta}$ is the shear or secondary wave ( $S$-wave). Considering the wave propagation of a plane wave along the $x_{1}$ axis, $u_{i}$ $=f\left(x_{1}-c t\right)=f\left(\tau_{1}\right)$, where $c$ denotes the wave velocity, Eq. (1) can be expressed as

$$
\rho c^{2} u_{1}^{\prime \prime}=(\lambda+2 \mu) u_{1}^{\prime \prime}, \rho c^{2} u_{2}^{\prime \prime}=\mu u_{2}^{\prime \prime} \text {, and } \rho c^{2} u_{3}^{\prime \prime}=\mu u_{3}^{\prime \prime} .
$$

Here, the primes denote differentiation with respect to $\tau_{1}$. The above equations can be also written as

$$
\rho \ddot{\mathbf{u}}=(\lambda+\mu) \nabla(\nabla \cdot \mathbf{u})+\mu \nabla^{2} \mathbf{u} .
$$

Employing the identities $\nabla^{2} \mathbf{u}=\nabla(\nabla . \mathbf{u})-\nabla \times(\nabla \times \mathbf{u})$ and $\rho \mathbf{u}_{t t}=(\lambda+2 \mu) \nabla(\nabla . \mathbf{u})-\mu \nabla \times(\nabla \times \mathbf{u})$, we next consider an axially symmetric compression wave in an infinite rod for which

$$
u_{r}=u_{r}(r, z, t), u_{z}=u_{z}(r, z, t) \text {, and } u_{\theta}=0 .
$$

The displacement equations for cylindrical waves provide the relations ${ }^{30}$

$$
\begin{gathered}
u_{r}=\partial \Pi / \partial r+1 / r \partial \psi / \partial \theta+l \partial^{2} \chi / \partial r \partial z \\
u_{\theta}=1 / r \partial \Pi / \partial \theta-\partial \psi / \partial r+l / r \partial^{2} \chi / \partial \theta \partial z,
\end{gathered}
$$

and

$$
u_{z}=\partial \Pi / \partial z-l\left[1 / r \partial / \partial r(r \partial \chi / \partial r)+1 / r^{2} \partial^{2} \chi / \partial \theta^{2}\right] .
$$

With $\psi=0$ in Eq. (7), the displacements are described through the relations

$u_{r}=\partial \Pi / \partial r+\partial^{2} \tilde{\chi} / \partial r \partial z$, and $u_{z}=\partial \Pi / \partial z-(1 / r) \partial / \partial r r \partial \tilde{\chi} / \partial r$,

where $\tilde{\chi}=l \chi$. The stresses involved in the boundary conditions are

$$
\sigma_{r r}=\lambda / c_{\alpha}^{2} \Pi_{, t t}+2 \mu\left(\partial^{2} \Pi / \partial r^{2}+\partial^{3} \tilde{\chi} / \partial r^{2} \partial z\right),
$$

and

$$
\sigma_{r z}=\mu\left[2 \partial^{2} \Pi / \partial r \partial z+\partial^{3} \tilde{\chi} / \partial r \partial z^{2}-\partial / \partial r(\partial / r \partial r(r \partial \tilde{\chi} / \partial r))\right] .
$$

We assume the solutions to these equations to be of the form

$$
\Pi=A J_{0}\left(\eta_{\alpha} r\right) e^{i(k z-\omega t)}, \text { and } \tilde{\chi}=B J_{0}\left(\eta_{\beta} r\right) e^{i(k z-\omega t)},
$$

where $\eta_{\alpha}^{2}=\omega^{2} / c_{\alpha}^{2}-k^{2}, \quad \eta_{\beta}^{2}=\omega^{2} / c_{\beta}^{2}-k^{2}$. The boundary conditions at the traction-free surface $r=a$ must equal zero, i.e., $\sigma_{r r}=\sigma_{r z}=0$. Substituting Eq. (10) into Eq. (9),

$$
\sigma_{r r}=\left[-\frac{\lambda \omega^{2}}{c_{\alpha}^{2}} J_{0}\left(\eta_{\alpha} r\right)+2 \mu \frac{d^{2} J_{0}\left(\eta_{\alpha} r\right)}{d r^{2}}\right] A+i 2 \mu k \frac{d^{2} J_{0}\left(\eta_{\beta} r\right)}{d r^{2}} B,
$$

and

$$
\begin{aligned}
\sigma_{r z}= & i 2 \mu k \frac{d J_{0}\left(\eta_{\alpha} r\right)}{d r} A \\
& -\left\{k^{2} \frac{d J_{0}\left(\eta_{\beta} r\right)}{d r}+\frac{d}{d r}\left[\frac{d}{r d r}\left(r \frac{d J_{0}\left(\eta_{\beta} r\right)}{d r}\right)\right]\right\} \mu B
\end{aligned}
$$

$A$ and $B$ are eliminated by taking the conditions at $r=a$ to obtain the Pochhammer equation, i.e.,

$$
\begin{aligned}
& 4 k^{2} \eta_{\alpha} \eta_{\beta} \mu J_{0}\left(\eta_{\beta} a\right) J_{1}\left(\eta_{\alpha} a\right) /\left(4 \pi^{2} \mu \eta_{\beta}\right)-2 \eta_{\alpha} \mu\left(k^{2}+\eta_{\beta}^{2}\right) \\
& \quad \times J_{1}\left(\eta_{\alpha} a\right) J_{1}\left(\eta_{\beta} a\right) /\left(4 \pi^{2} a \mu \eta_{\beta}\right)+\left(-k^{2}+\eta_{\beta}^{2}\right) J_{0}\left(\eta_{\alpha} a\right) \\
& \quad \times J_{1}\left(\eta_{\beta} a\right)\left(\lambda \omega^{2}+2 \eta_{\alpha}^{2} \mu c_{\alpha}^{2}\right) /\left(4 \pi^{2} \mu \eta_{\beta} c_{\alpha}^{2}\right)=0 \\
& \quad \text { or }\left(k^{2}-\eta_{\beta}^{2}\right)^{2} \frac{\alpha J_{0}\left(\eta_{\alpha} a\right)}{J_{1}\left(\eta_{\alpha} a\right)}+4 k^{2} \eta_{\alpha}^{2} \frac{\beta J_{0}\left(\eta_{\beta} a\right)}{J_{1}\left(\eta_{\beta} a\right)} \\
& \quad-2 \eta_{\alpha}^{2}\left(\eta_{\beta}^{2}+k^{2}\right)=0
\end{aligned}
$$

Next, we consider the nanowire under surface stress (tension) to be governed by the Euler-Bernoulli beam equation, ${ }^{28}$

$$
\partial_{t, t} u_{z}=[(E / \rho)-2 v \sigma /(\rho R)] \partial_{z, z} u_{z},
$$

where $E$ denotes the Young's modulus, $R$ the nanowire radius, and $\sigma$ the surface stress. The value of $u_{z}$ obtained from Eq. (8) must satisfy Eq. (13), or,

$$
\frac{\left(k^{2} a E-2 k^{2} v \sigma-a \rho \omega^{2}\right)\left[2 i k a J_{0}\left(\eta_{\alpha} a\right)\right]}{2 \rho a^{2}} A+\frac{\left(k^{2} a E-2 k^{2} v \sigma-a \rho \omega^{2}\right)\left[a \eta_{\beta}^{2} J_{0}\left(\eta_{\beta} a\right)+2 \eta_{\beta} J_{1}\left(\eta_{\beta} a\right)-a \eta_{\beta}^{2} J_{2}\left(\eta_{\beta} a\right)\right]}{2 \rho a^{2}} B=0 .
$$


where $R=a$ and $\sigma_{r r}$ vanishes for the free-surfaces at the ends. Hence,

$$
\begin{aligned}
\sigma_{r r}= & \left(-\left[\lambda \omega^{2} J_{0}\left(\eta_{\alpha} a\right) /\left(c_{\alpha}^{2}\right)\right]+2 \mu\left\{-(1 / 2) \eta_{\alpha}^{2}\left[J_{0}\left(\eta_{\alpha} a\right)\right.\right.\right. \\
& \left.\left.\left.-J_{2}\left(\eta_{\alpha} a\right)\right]\right\}\right) A-\left\{i k \mu \eta_{\beta}^{2}\left[J_{0}\left(\eta_{\beta} a\right)-J_{2}\left(\eta_{\beta} a\right)\right]\right\} B=0 .
\end{aligned}
$$

Based on Eqs. (14) and (15), we obtain the dispersion relation,

$\eta_{\beta}\left(k^{2} a E-2 k^{2} v \sigma-\rho a \omega^{2}\right)\left\{2 c_{\alpha}^{2} \eta_{\alpha} \eta_{\beta} \mu J_{0}\left(\eta_{\beta} a\right) J_{1}\left(\eta_{\alpha} a\right)\right.$

$-J_{0}\left(\eta_{\alpha} a\right) \times\left[a \eta_{\beta}\left(2 c_{\alpha}^{2}\left(k^{2}+\eta_{\alpha}^{2}\right) \mu+\lambda \omega^{2}\right) J_{0}\left(a \eta_{\beta}\right)\right.$

$\left.\left.-2 c_{\alpha}^{2} k^{2} \mu J_{1}\left(a \eta_{\beta}\right)\right]\right\} /\left(c_{\alpha}^{2} a^{2} \rho\right)=0$.

Defining, $k^{*}=k a, \omega^{* 2}=\rho a^{2} \omega^{2} / \mu, \alpha=\eta_{\alpha} a$, and $\beta=\eta_{\beta} a$ in terms of dimensionless parameters, Eq. (16) assumes the form,

$$
\begin{aligned}
& \left\{\beta k^{* 2}[(E / \mu)-(2 v \sigma / \mu a)]-\beta \omega^{* 2}\right\} \\
& \left\{\begin{array}{l}
2 \alpha \beta J_{0}(\beta) J_{1}(\alpha)-J_{0}(\alpha) \\
{\left[\left(2 k^{* 2} \beta+2 \beta \alpha^{2}+\left(\lambda \beta \omega^{* 2}\right) /\left(\rho c_{\alpha}^{2}\right)\right) J_{0}(\beta)-2 k^{* 2} J_{1}(\beta)\right]}
\end{array}\right\}=0 .
\end{aligned}
$$

\section{B. Calculation of the lattice thermal conductivity}

Acoustic phonon relaxation can be modeled in resistive processes by considering three-phonon Umklapp scattering, mass-difference scattering, boundary scattering, and phononelectron scattering. ${ }^{31}$ The combined scattering relaxation rate can be determined from Matthiessen's rule, ${ }^{3}$

$$
1 / \tau_{c}=1 / \tau_{U}+1 / \tau_{M}+1 / \tau_{B}+1 / \tau_{P h-e},
$$

where $1 / \tau_{U}, 1 / \tau_{M}, 1 / \tau_{P h-e}$, and $1 / \tau_{B}$ are the phonon relaxation rates for the three-phonon Umklapp, mass-difference (due to impurities), phonon-electron, and boundary scattering processes, respectively.

The Umklapp scattering rate,

$$
1 / \tau_{U}=2 \gamma^{2} k_{B} T \omega^{2} /\left(\mu V_{0} \omega_{D}\right),
$$

where $\gamma$ denotes the Grüneisen anharmonicity parameter, $\mu$ the shear modulus, $V_{0}$ the volume per atom and $\omega_{D}$ the Debye frequency. The phonon relaxation rate for point defects,

$$
1 / \tau_{M}=V_{0} \omega^{4} \Gamma /\left(4 \pi V^{3}\right)=V_{0} \omega^{4} /\left(4 \pi V^{3}\right) \sum_{i} f_{i}\left(1-M_{i} / \bar{M}\right)^{2},
$$

where $\Gamma$ denotes the measure of the strength of the pointdefect scattering, $V$ the phonon group velocity, $f_{i}$ the relative concentration of the $i$ th atoms, $\bar{M}=\sum_{i} f_{i} M_{i}$ the average atomic mass, and $M_{i}$ the mass of the $i$-th impurity atom. The relaxation frequency for the intravalley and intervalley interactions between electrons and phonons,

$$
\begin{aligned}
1 / \tau_{P h-e}= & \sqrt{\pi m^{*} V^{2} /\left(2 k_{B} T\right)} \\
& \times \exp \left[-m^{*} V^{2} /\left(2 k_{B} T\right)\right] n_{e} \varepsilon_{1}^{2} \omega /\left(\rho V^{2} k_{B} T\right),
\end{aligned}
$$

where $m^{*}$ denotes the electron effective mass, $n_{e}$ the concentration of conduction electrons, and $\varepsilon_{1}$ the deformation potential. The frequency based on the geometrical sample size is

$$
1 / \tau_{B}=V(1-p) / D
$$

where $D$ denotes the nanowire diameter, and $p$ describes the probability that a phonon is subjected to a specular scattering at the interface. ${ }^{31}$ It characterizes boundary scattering effects due to the interface roughness. The boundary scattering rate is zero when there is purely specular scattering, or $p=1$, and for purely diffuse scattering $p=0$. Purely specular scattering $(p=1)$ refers to an idealized case when all phonons travel through the nanowire without any boundary reflections. For $p=0$, all phonons are scattered at the interface.

The Klemens-Callaway's expression provides the lattice thermal conductivity in a nanowire,

$$
\kappa_{l}=\left(k_{B} T / \hbar\right)^{3}\left[k_{B} /\left(2 \pi^{2} V\right)\right] \int_{0}^{\theta_{D} / T} \tau_{c} x^{4} e^{x} /\left(e^{x}-1\right)^{2} d x,
$$

where $k_{B}$ denotes the Boltzmann constant, $\hbar$ the Planck constant, $T$ the temperature, $\theta_{D}$ the Debye temperature, $\tau_{c}$ the combined phonon relaxation time derived from Eq. (18), and $x=\hbar \omega / k_{B} T$. To characterize the influence of the interface roughness on the phonon-boundary scattering, $p$ represents the probability that the phonon is subject to a specular scattering event at the interface. Hence, (1$p$ ) is the probability that a phonon undergoes diffuse scattering. Thus, for a nanowire, the lattice thermal conductivity $\kappa_{l}^{\text {wire }}$ can be expressed in terms of the bulk thermal conductivity $\kappa_{l}$ as, ${ }^{31}$

$$
\kappa_{l}^{\text {wire }}(T, p)=\kappa_{l}(T, p)-\Delta \kappa_{l}^{\text {wire }}(T, p) .
$$

The deviation of the thermal conductivity from its bulk value,

$\Delta \kappa_{l}^{\text {wire }}=12\left(k_{B} T / \pi \hbar\right)^{3} k_{B} / V \int_{0}^{\theta_{D} / T} \tau_{c} x^{4} e^{x} G[\eta(x), p] /\left(e^{x}-1\right)^{2} d x$,

where $\eta$ denotes the ratio between the wire diameter $D$ and the phonon mean free path $\Lambda$, i.e.,

$$
\eta(x)=D / \Lambda(x), \text { with } \Lambda(x)=V(x) \tau_{c}(x) .
$$

The function

$$
G[\eta(x), p]=(1-p)^{2} \sum_{j=1}^{\infty} j p^{j-1} \int_{0}^{1}\left(1-y^{2}\right)^{1 / 2} S_{4}(j \eta y) d y,
$$

where

$$
S_{n}(u)=\int_{0}^{\pi / 2} e^{-u / \sin \theta} \cos ^{2} \theta \sin ^{n-3} \theta d \theta
$$


Substituting Eqs. (25)-(28) in Eq. (23) and using Eq. (24),

$$
\begin{aligned}
\kappa_{l}^{\text {wire }}(T, p)= & \left(k_{B} T / \hbar\right)^{3} k_{B} /\left(2 \pi^{2} V\right) \int_{0}^{\theta_{D} / T} \tau_{c} x^{4} e^{x} /\left(e^{x}-1\right)^{2} \\
& \times\{1-24 G[\eta(x), p] / \pi\} d x .
\end{aligned}
$$

For purely specular scattering, $p \rightarrow 1$ and $G \rightarrow 0$. Hence, $\kappa_{l}$ equals the thermal conductivity from by Eq. (24) with $\Delta \kappa_{l}^{\text {wire }}=0$. Likewise, $\Delta \kappa_{l}^{\text {wire }} \rightarrow 0$ as the wire diameter $D \rightarrow \infty$.

\section{RESULTS AND DISCUSSIONS}

We select nanowires of 2 and $4 \mathrm{~nm}$ diameters to investigate surface stress effects on phonon dispersion. While silicon pillars as small as $2 \mathrm{~nm}$ in diameter have been successfully fabricated using stress-limited oxidation, ${ }^{32}$ a nanowire of such small dimensions is not typically freestanding. Our intention in proposing an ultrathin nanowire is to exhibit the interesting influence of surface stress on thermal conductivity, e.g., on a thin surface coated with another material (that induces surface stress), which helps in conceptualizing and clarifying stress effects.

The material parameters for a semiconductor nanowire are taken to be $c_{\alpha}=8.47 \times 10^{3} \mathrm{~m} \mathrm{~s}^{-1}$, and $c_{\beta}=5.34 \times 10^{3}$ $\mathrm{m} \mathrm{s}^{-1}$ (Ref. 31). The Young's modulus has been reported to decrease from $90 \mathrm{GPa}$ for bulk $\langle 100\rangle$ silicon to $18 \mathrm{GPa}$ for silicon nanowires that have diameters smaller than $10 \mathrm{~nm}^{33}$ Thus, we use $E=18 \mathrm{GPa}$ in the dispersion relations for 2 and $4 \mathrm{~nm}$ diameter nanowires. Figure 1 presents this relation for acoustic phonons in a freestanding $2 \mathrm{~nm}$ diameter silicon nanowire. The dispersion is linear for very small values of $k$ for the first branch. This is called the acoustic branch since its dispersion relation has the form $\omega=c k$ for small $k$, where $c$ denotes the sound velocity. Here, the group velocity of the first branch (or the slope of the curve at small values of phonon energy) nearly coincides with the sound velocity in the bulk material. Each phonon dispersion branch corresponds to a longitudinal vibration mode for the nanowire.

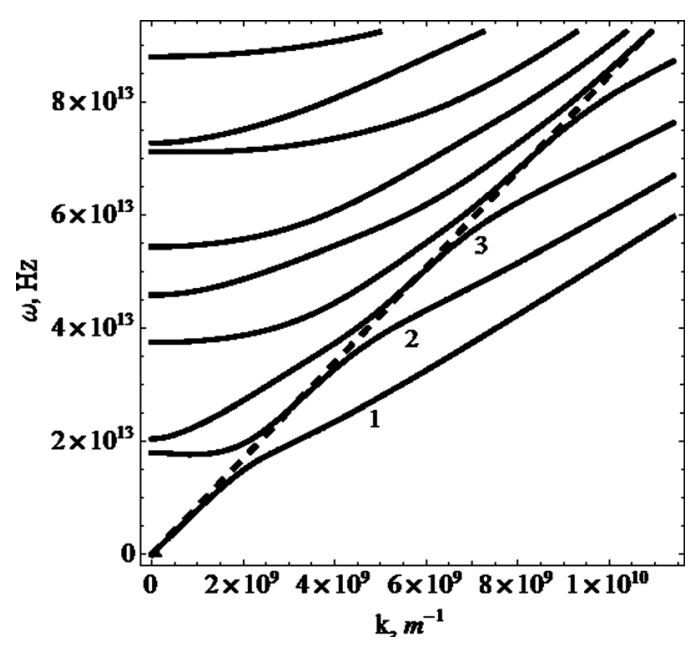

FIG. 1. Acoustic phonon dispersion relation in a free-standing $2 \mathrm{~nm}$ diameter silicon nanowire. The dotted line denotes the linear (bulk) dispersion relation for the longitudinal polarization.
Figure 2 presents acoustic phonon dispersion relations for silicon nanowires with and without surface stress. Wires of $2 \mathrm{~nm}$ diameters are considered in Figs. 2(a) and 2(b) and of $4 \mathrm{~nm}$ diameter in Figs. 2(c) and 2(d). We compare the influences of $1 \mathrm{~N} \mathrm{~m}^{-1}$ and $5 \mathrm{~N} \mathrm{~m}^{-1}$ surface stresses for nanowires of both diameters. The first two branches show linear dispersions for all values of $k$ when the nanowire is stressed (represented by solid lines). Increasing the surface stress reduces the energy associated with the first acoustic branch. Changing the surface stress for the larger diameter nanowire does not diminish the energy as significantly. This can be explained by considering the decrease in the surface area to volume ratio as the characteristic object size increases, which greatly decreases the global influence of the surface stress. These results also show that phonon dispersion can be modified through surface stress, more significantly so for nanowires of smaller diameters.

A thermal resistance is produced when the phonon momentum is not conserved. This can occur through phonon scattering due to (1) impurities, defects, boundary surfaces, and electrons, and (2) phonon-phonon Umklapp processes when two phonons merge into one or a single phonon splits into two. Since longitudinal phonons usually contain more energy than transverse ones, the former can decay either into a longitudinal and a transverse phonon, or into two transverse phonons. The resistance to thermal transport can be created through three such Umklapp processes. ${ }^{34}$ These are described based on the phonon polarization, i.e., $T+T \leftrightarrow L$, $T+L \leftrightarrow L$, and $L+T \leftrightarrow L$, where $L$ and $T$ refer to the longitudinal and transverse acoustic branches. ${ }^{34}$ Such a mechanism has been theoretically predicted in zigzag single-walled carbon nanotubes. ${ }^{35}$

Since a single phonon can split into two phonons, the longitudinal acoustic phonons in a stressed nanowire should also behave similarly. The shift in the first branch in Fig. 2 suggests that the phonon Umklapp scattering lowers the energy (or frequency) as the surface stress increases. However, as the nanowire diameter increases, phonon boundary scattering over the larger surface area dominates over Umklapp scattering. This is consistent with the literature which reports that the phonon dispersion relation of a silicon nanowire can be modified for diameter size below $10 \mathrm{~nm}$ due to reduced phonon group velocities and phonon confinement. ${ }^{6,7,36}$

Equation (19) shows that the scattering rate due to Umklapp process is inversely proportional to the Debye temperature. When the phonon dispersion relation is modified, it changes this temperature. The cutoff wave number $q_{D}$ for an isotropic crystal with a lattice constant $a$ is given by $4 \pi q_{D}^{3} / 3=4(2 \pi)^{3} / a^{3}$ (Ref. 37). We employ a lattice constant of $5.43 \AA$ for the Si nanowire. The corresponding Debye frequency $\omega_{D}$, which is the maximum allowed vibration frequency for atoms in a crystal, is determined by using $q_{D}$ in the phonon dispersion relations. The Debye temperatures $\theta_{D}=\hbar \omega_{D} / k_{B}$ for the $2 \mathrm{~nm}$ diameter nanowire are, respectively, $417 \mathrm{~K}$ and $222 \mathrm{~K}$ for the stress free case and for a $5 \mathrm{~N} \mathrm{~m}^{-1}$ surface stress. Hence, $\theta_{D}$ is altered when the phonon dispersion relation is modified by surface stress. The lattice thermal conductivity calculated from 


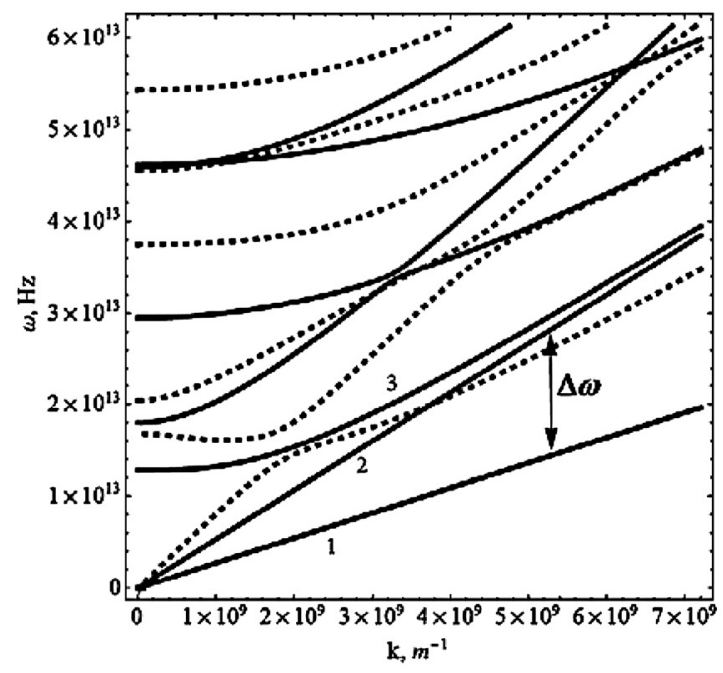

(a)

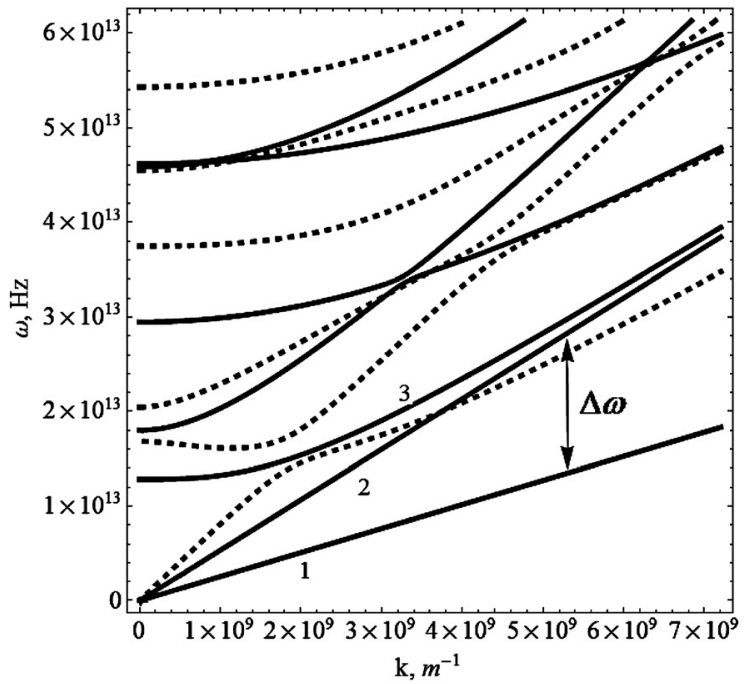

(b)

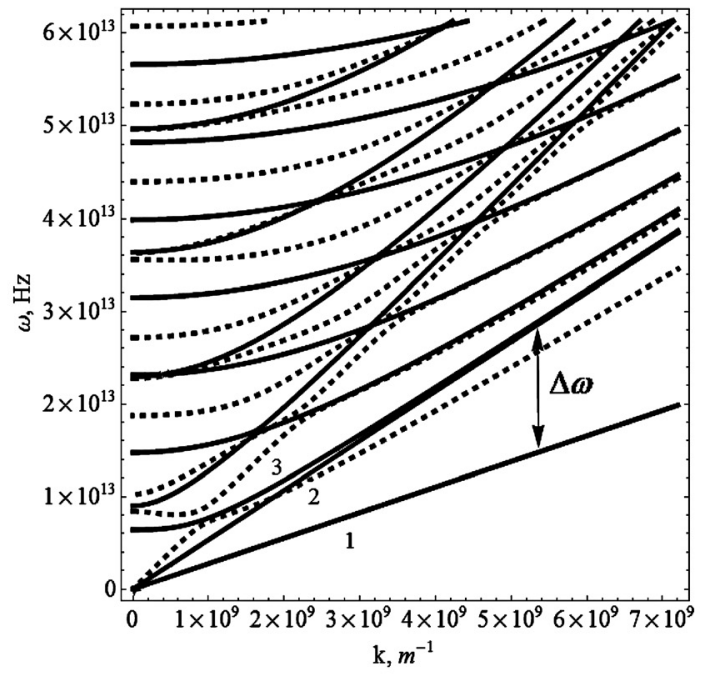

(c)

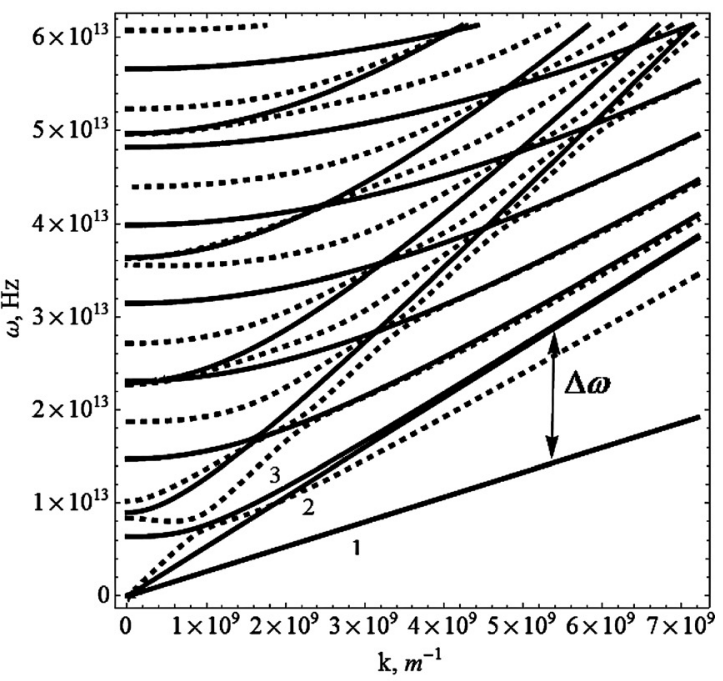

(d)

FIG. 2. Acoustic phonon dispersion relations for silicon nanowire that are stress free (dashed lines) and subject to surface stress (solid lines). A 2 nm diameter nanowire is subject to (a) 1 , (b) $5 \mathrm{~N} \mathrm{~m}^{-1}$ surface stresses, respectively. Similarly, a $4 \mathrm{~nm}$ diameter nanowire is subject to (c) 1 , (d) $5 \mathrm{~N} \mathrm{~m}^{-1}$ stresses.

Eqs. (23)-(25) is therefore also expected to decrease as $\theta_{D}$ is reduced.

From Eq. (17), the difference $E / \mu-2 v \sigma / \mu a$ significantly influences the first branch of the dispersion relation. Of these parameters, only $\sigma$ can be varied once a material and its dimensions are specified. The ratio $E / \mu$ compares the Young's modulus to the phonon pressure $\mu=\rho c_{\beta}^{2}$. A phonon is the quanta of energy associated with the vibrating atoms. The phonon frequency, thus the thermal energy, decreases when the volume of a material increases. While the phonon pressure tends to expand the material, its lattice is held together by internal forces (for which $E$ is a measure of the material stiffness). The term $2 v \sigma / \mu a$ compares the work done to stretch a unit area of the nanowire surface to that done by the acoustic phonons that travel transversely across the cross section of the nanowire. Another term in Eq. (17), $\lambda \beta \omega^{*^{2}} /\left(\rho c_{\alpha}^{2}\right)$ can be simplified as $\lambda \beta \omega^{* 2} /(\lambda+2 \mu)$ for which $\lambda /(\lambda+2 \mu)$ is related to the material properties alone.
Figure 3 presents the dispersion relations for a silicon nanowire in terms of the dimensionless parameters $k^{*}$ and $\omega^{*}$. Three cases for which ratio $2 v \sigma / \mu a$ is taken to be 0 , 0.05 , and 0.1 , respectively, are represented by the dotted, solid, and dashed lines, whereas $E / \mu=0.2$ for all cases. Varying the surface stress through $2 v \sigma / \mu a$ only influences the first branch notably. Increasing stress reduces the phonon energy for this branch. The averaged group velocity for all branches is smaller when the nanowire is subject to a larger stress because of the reduction in the slope of the first branch. As the surface stress increases, the energy difference between the first and the second phonon dispersion branches becomes larger, since the first branch now is associated with lower energy.

The lattice thermal conductivity can be calculated once the relationship between the phonon group velocity and phonon frequency is known. The averaged phonon group velocity can be determined by differentiating the dispersion 


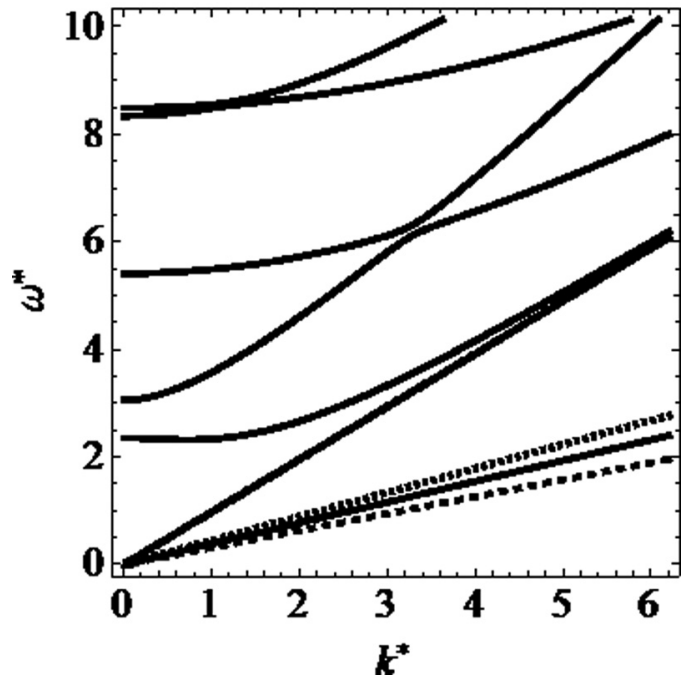

FIG. 3. Dispersion relation for a silicon nanowire in terms of $k^{*}$ and $\omega^{*}$. The ratio $2 v \sigma / \mu a$ is $0,0.05$ and 0.1 , respectively, for the dotted, solid, and dashed lines while $E / \mu=0.2$ for all cases.

relations. This can be used to determine the relaxation times for mass difference and boundary scattering as well as for acoustic phonons scattered by electrons from Eqs. (20)-(22). Since each branch has different group velocities at different energies, contributions from all branches are required to calculate the average group velocity corresponding to a specific frequency $\omega$, namely, ${ }^{37}$

$V(\omega)=\left[\sum_{n} V_{n} \exp \left(-n \hbar \omega / k_{B} T\right)\right] /\left[\sum_{n} \exp \left(-n \hbar \omega / k_{B} T\right)\right]$.

Here, the group velocity of the $\mathrm{n}^{\text {th }}$ mode $V_{n}=d \omega_{n} / d k$

As shown in Fig. 4, the averaged group velocities for a silicon nanowire that does not experience surface stress are almost identical at small frequencies to the bulk material velocity $c_{\alpha}=8,470 \mathrm{~m} \mathrm{~s}^{-1}$. As the frequency (or energy) increases, the group velocity for the stress-free nanowire first abruptly decreases and then increases before exhibiting a

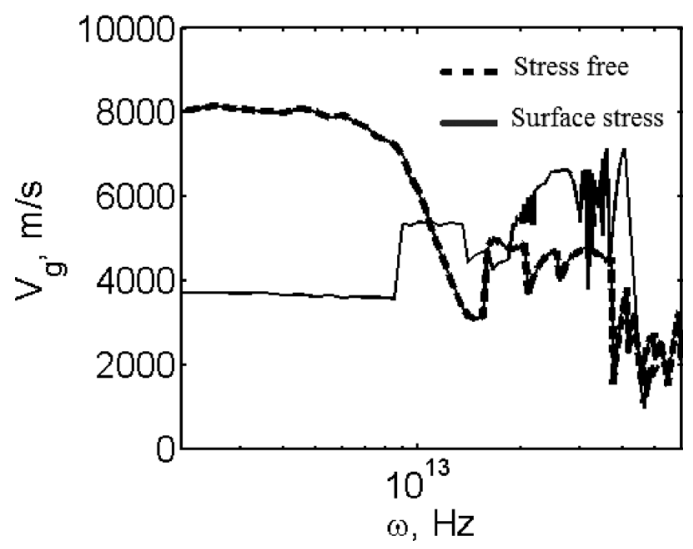

FIG. 4. Phonon group velocity as a function of phonon frequency (in log scale) for a $2 \mathrm{~nm}$ diameter silicon nanowire. The dashed line represents the surface stress free condition and the solid line when a $5 \mathrm{~N} \mathrm{~m}^{-1}$ surface stress is imposed upon the nanowire. gradual decline. The group velocities for both stressed and stress-free nanowires are almost constant at smaller frequencies. Unsurprisingly, those for the nanowire with a $5 \mathrm{~N} \mathrm{~m}^{-1}$ stress are smaller, reflecting the decrease in the lattice thermal conductivity. However, unlike for the stress free nanowire, the group velocities for nanowire experience stress increase and fluctuate around $5000 \mathrm{~m} \mathrm{~s}^{-1}$, eventually decreasing to $2000 \mathrm{~m} \mathrm{~s}^{-1}$. This final value is below the transverse velocity $c_{\beta}=5,340 \mathrm{~m} \mathrm{~s}^{-1}$ in the bulk silicon. ${ }^{31}$ This reflects how surface stress alters the phonon waves inside the nanostructure.

Once the group velocities are known, phonon scattering rates can be evaluated from Eqs. (19)-(22). The material parameters taken from the literature for this calculation are listed in Table I. The scattering rates due to the three-phonon Umklapp, mass difference, boundary (for which the case of purely diffuse scattering, or $p=0$ is considered), and phonon-electron scattering mechanisms are expressed as a function of phonon frequency. The dominant low frequency mechanism is boundary scattering $\left(1 / \tau_{B}\right)$. The mass difference and Umklapp scattering both increase significantly at higher frequencies. Among these mechanisms, phonon-electron scattering rates are relatively small. Changes in the averaged group velocities for the stress free and stressed conditions also influence these differences in the scattering rates. Overall, a reduction in the group velocity decreases phonon relaxation, thus leading to a reduction in the lattice thermal conductivity.

Next, the parameter $p$ is varied between zero and unity to characterize the influence of boundary scattering on the lattice thermal conductivity. For instance, a rougher boundary has a smaller $p$ value than a smoother one. The variation in the lattice thermal conductivity with changing $p$ is presented in Fig. 5 at $300 \mathrm{~K}$. When $p=0$, this conductivity decreases when a $5 \mathrm{~N} \mathrm{~m}^{-1}$ surface stress is imposed to about $75 \%$ of its corresponding stress free value. As $p$ increases, the thermal conductivities in the stress free and stressed nanowires both increase. However, influence of surface stress diminishes with increasing $p$ so that $\Delta \kappa_{l}^{\text {wire }}$ decreases. The two (stress free and surface stress related) conductivities are virtually identical when $p=1$, i.e., $\Delta \kappa_{l}^{\text {wire }} \approx 0$. This is intuitive, since increasing $p$ decreases the role of boundary

TABLE I. Parameters used for the phonon scattering rate calculation for a silicon nanowire. ${ }^{3,31}$

\begin{tabular}{ll}
\hline \hline Parameters & Values \\
\hline Number of atoms per unit volume $n_{a}$ & $\approx 7.3$ \\
Atomic mass $M(\mathrm{~kg})$ & $46.6 \times 10^{-27}$ \\
Grüneisen parameter $\gamma$ & 0.56 \\
Lattice constant $a($ Angstrom $)$ & 5.45 \\
Crystal density $\rho\left(\mathrm{kg} \mathrm{m}^{-3}\right)$ & $2.33 \times 10^{3}$ \\
Strength of the mass-difference scattering $\Gamma$ & $8.357 \times 10^{-4}$ \\
Shear modulus $\left(\mu=v_{t}^{2} \rho\right),(\mathrm{GPa})$ & 66.4 \\
Deformation potential $\varepsilon_{1}(\mathrm{eV})$ & 9.5 \\
Concentration of conduction electrons $n_{e}\left(\mathrm{~cm}^{-3}\right)$ & $10^{18}$ \\
Boltzmann constant $k_{B}\left(\mathrm{~m}^{2} \mathrm{~kg} \mathrm{~s}^{-2} \mathrm{~K}^{-1}\right)$ & $1.38 \times 10^{-23}$ \\
Young's modulus $E(\mathrm{GPa})$ & 18 \\
\hline \hline
\end{tabular}




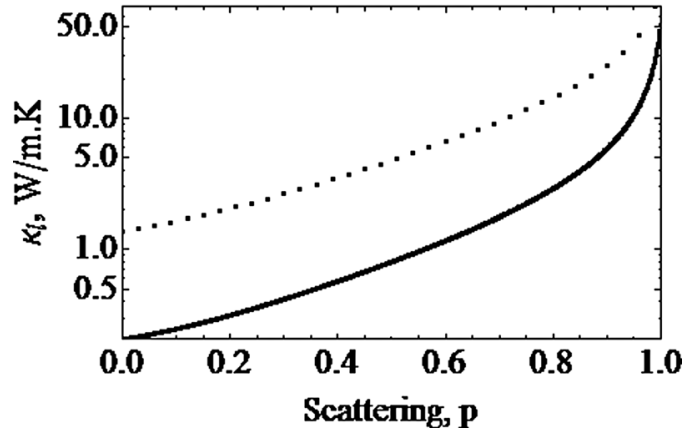

FIG. 5. The variation in the lattice thermal conductivity for a $2 \mathrm{~nm}$ diameter silicon nanowire that is stress free (dotted line) and under a $5 \mathrm{~N} \mathrm{~m}^{-1}$ surface stress (solid line) of the specular fraction $p$ varied at $T=300 \mathrm{~K}$.

scattering so that boundary effects such as surface stress become less important.

Once the phonon group velocity, the various relaxation times, and the Debye temperature and frequency are known, the lattice thermal conductivity can be calculated. Equations (20) and (22), show that a reduction in the averaged group velocity increases the mass difference scattering rate and decreases the boundary scattering rate. A reduction in the Debye frequency $\left(\omega_{D}\right)$ increases the Umklapp scattering rate [Eq. (19)], thus decreases the phonon relaxation time. Therefore, phonon dispersion is influenced by surface stress not only through a decrease in the averaged phonon group velocity, but also by a decrease in the Debye frequency and Debye temperature. All of these effects combine to reduce the thermal conductivity.

For sake of illustration, Fig. 6(a) presents the variation in the thermal conductivity with changing temperature due to the Umklapp, mass difference and phonon-electron scattering mechanisms based on Eqs. (19)-(22). We again assume that a $2 \mathrm{~nm}$ diameter silicon nanowire is either stress free or experiences a $5 \mathrm{~N} \mathrm{~m}^{-1}$ surface stress, and there is completely diffuse scattering, i.e., $p=0$ because of the very small diameter nanowire and the potential irregularities at the interface due to the surface stress. The thermal conductivities due to Umklapp scattering are the largest contributors for both conditions and decrease with increasing temperature. At $300 \mathrm{~K}$, the thermal conductivity due to Umklapp processes drops from $\approx 55 \mathrm{~W} \mathrm{~m}^{-1} \mathrm{~K}^{-1}$ (for the stress free case) to $\approx 28 \mathrm{~W} \mathrm{~m}^{-1} \mathrm{~K}^{-1}$ (for the nanowire with surface stress). This stems from the reduction in the Debye temperature that leads to a smaller phonon relaxation time, as is apparent from Eq. (19). Adding mass-difference scattering to the combined relaxation time $\tau_{c}$ decreases the overall conductivity, which is further diminished when phonon-electron scattering is also accounted for.

The associated lattice thermal conductivities for all mechanisms are presented in Fig. 6(b) when the boundary scattering is included. The dominant mechanism here is boundary scattering. It significantly reduces the thermal conductivity below the values attributed to the three mechanisms discussed in Fig. 6(a) by $\approx 20-50 \mathrm{~W} \mathrm{~m}^{-1} \mathrm{~K}^{-1}$ to values smaller than $2 \mathrm{~W} \mathrm{~m}{ }^{-1} \mathrm{~K}^{-1}$. Thus, boundary scattering decreases the thermal conductivity by one and two orders of magnitude for the stress free and stressed nanowires, respec-

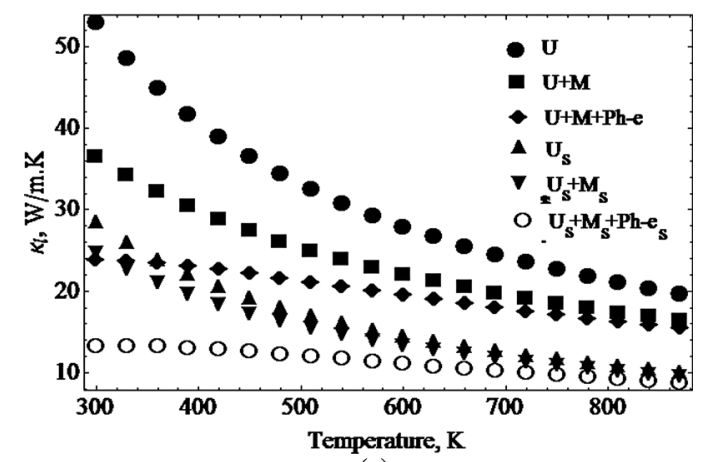

(a)

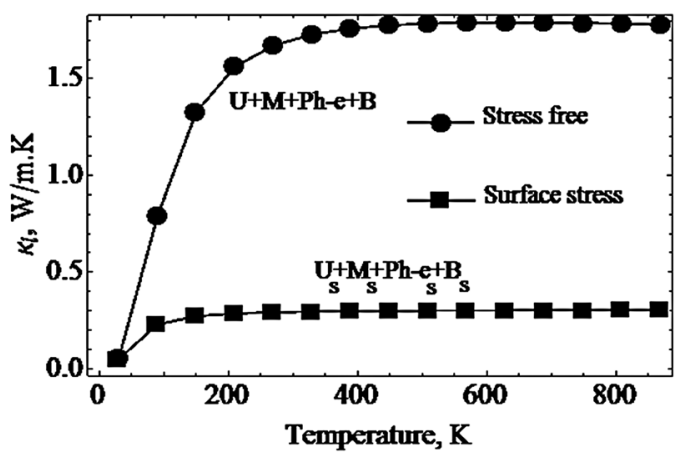

(b)

FIG. 6. (a) Lattice thermal conductivities for $2 \mathrm{~nm}$ silicon nanowires that are stress free and experience surface stress (denoted by the subscript $\mathrm{s}$ ) when different scattering mechanisms are considered for purely diffuse scattering, i.e., $p=0$. The subscripts $U, M$, and $P h-e$ denote the Umklapp, massdifference (due to impurities), and phonon-electron processes. (b) The thermal conductivity calculated with $p=0$ and after considering the $U, M, P h-e$, and $B$ (boundary scattering) mechanisms for these nanowires.

tively. It is important to consider all scattering events considered in Eq. (18) to determine the combined phonon relaxation time $\left(\tau_{c}\right)$ before calculating the thermal conductivity from Eq. (29). The corresponding thermal conductivity is proportional to the integrated value of $\tau_{c}$ from zero to the upper limit that is the ratio between the Debye temperature and room temperature $(300 \mathrm{~K})$. Hence, decreasing $\tau_{c}$ results in a reduction in the overall thermal conductivity.

Figure 6(b) presents the lattice thermal conductivity as a function of temperature when $p=0$. The conductivity of the stressed nanowire increases with temperature but less significantly $\approx 300 \mathrm{~K}$. The imposition of $5 \mathrm{~N} \mathrm{~m}^{-1}$ surface stress produces a large decrease in the thermal conductivity over the entire temperature range, which is a significant result. Our analysis can also be used to evaluate surface stress effects on the lattice thermal conductivity of a thin film. Surface stress can be generated on a clean surface or a thin film system, such as a nanowire, by coating it. The magnitude of the stress can be varied by selecting specific coating materials that induce different forces on the surface. Figure 3 shows that for a given nanostructure, the larger the surface stress is, the more it decreases the phonon frequency for a specified wavenumber. Thus, differing magnitudes of the stress produce different changes in the averaged group velocity and Debye temperature, leading to variations in the thermal conductivity as a function of stress.

Molecular dynamics (MD) simulations ${ }^{38}$ have predicted the temperature dependence of the thermal conductivity of 


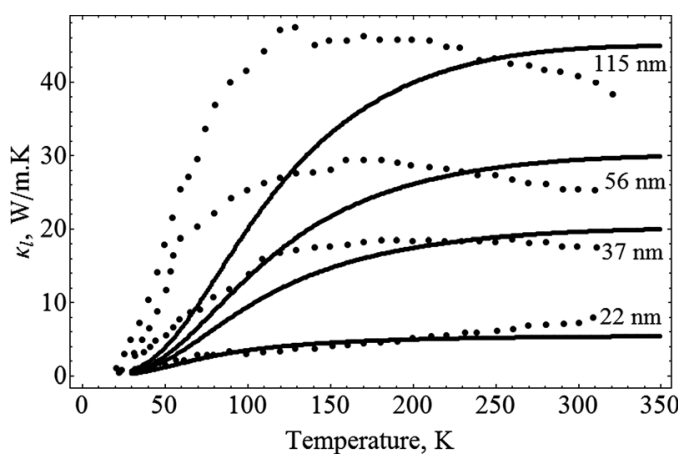

FIG. 7. Thermal conductivity of different diameter silicon nanowires. Dotted lines represent the previously experimentally measured thermal conductivity. ${ }^{36}$ Solid lines are predictions from our model results.

crystalline silicon and silicon core-shell nanowires that are 2$3 \mathrm{~nm}$ in diameter and compared with results obtained from the Boltzmann transport equation (BTE), and Allen and Feldmann (AF) theory. ${ }^{38}$ The thermal conductivity predicted by all these approaches has values between $2-5 \mathrm{~W} \mathrm{~m}^{-1} \mathrm{~K}^{-1}$. Our results, presented in Fig. 6(b) for $p=0$ at $300 \mathrm{~K}$, are somewhat different. This is most likely due to the different values of various material parameters used to calculate the thermal conductivity, which for our case are reported in Table I. Since values of some parameters are not reported in Ref. 38 , and since it is also possible that only some phonon relaxations were taken into account by that investigation, it is difficult to pinpoint the exact source of the discrepancy. Selecting $p=0.6$ at $300 \mathrm{~K}$, the lattice thermal conductivity predicted by our model, shown in Fig. 5, varies between 1-7 $\mathrm{W} \mathrm{m}^{-1} \mathrm{~K}^{-1}$ for two cases, one with a $5 \mathrm{~N} \mathrm{~m}^{-1}$ surface stress and the other stress free. While this range is wide, it approximates the results obtained by MD, BTE, and AF + BTE. $^{38}$

Figure 7 compares the thermal conductivities for 22, 37, 56 , and $115 \mathrm{~nm}$ diameter wires with experimental measurements. ${ }^{36}$ Our model assumes a $5 \mathrm{~N} \mathrm{~m}^{-1}$ surface stress and purely diffuse scattering $(p=0)$. As mentioned earlier, the Young's modulus varies between 18 and $90 \mathrm{GPa}$ for silicon nanowires. ${ }^{33}$ Therefore, we assume $E=32,60,80$, and 80 $\mathrm{GPa}$ for the 22, 37, 56, and $115 \mathrm{~nm}$ diameter nanowires, respectively. The thermal conductivities of the smaller (22 and $37 \mathrm{~nm}$ ) of these nanowires are in good agreement with experiments. However, discrepancies arise for the larger diameter nanowires because the major contributor to heat transport at these dimensions is now volumetric so that the influence of surface atoms diminishes. Thus, as is intuitive, surface stress has a much more significant influence on phonon transport for smaller diameter nanowires.

\section{CONCLUSION}

Surface stress arises when thin films or surface atoms undergo dynamic structural changes, e.g., such as those in the density of atoms attached to a substrate. However, even a clean surface or a thin film can be subject to a stress that is generated from the redistributed charge density on its surface. We investigate the effect of surface stress on the lattice thermal conductivity in a silicon nanowire. Surface stress strongly influences the phonon dispersion although its effect diminishes in significance as the nanowire diameter increases. An applied surface stress reduces the averaged group velocities of phonons and the Debye temperature, thus increasing Umklapp phonon scattering and decreasing the overall phonon relaxation time. Phonon scattering also depends upon its specular fraction $p$, and decreases with increasing $p$. When surface stress is applied on a silicon nanowire, it reduces the material lattice thermal conductivity. This finding can be used for phonon engineering approaches that seek enhanced thermal management in nanostructures, and to optimize thermoelectric devices.

${ }^{1}$ H. S. Park, J. Appl. Phys. 103, 123504 (2008).

${ }^{2}$ X. Lu and J. Chu, J. Appl. Phys. 100, 014305 (2006).

${ }^{3}$ X. Lu, J. H. Chu, and W. Z. Shen, J. Appl. Phys. 93, 1219 (2003).

${ }^{4}$ G. D. Mahan and J. O. Sofo, Proc. Natl. Acad. Sci. U.S.A. 93, 7436 (1996).

${ }^{5}$ E. P. Pokatilov, D. L. Nika, and A. A. Balandin, Superlattices Microstruct. 38, 168 (2005).

${ }^{6}$ A. Balandin and K. L. Wang, Phys. Rev. B 58, 1544 (1998).

${ }^{7}$ A. Khitun, A. Balandin, and K. L. Wang, Superlattices Microstruct. 26, 181 (1999).

${ }^{8}$ E. P. Pokatilov, D. L. Nika, and A. A. Balandin, Phys. Rev. B 72, 113311 (2005).

${ }^{9}$ E. P. Pokatilov, D. L. Nika, and A. A. Balandin, Superlattices Microstruct. 33, 155 (2003).

${ }^{10}$ G. Balasubramanian, S. Banerjee, and I. K. Puri, J. Appl. Phys. 104, 064306 (2008).

${ }^{11}$ S. Murad and I. K. Puri, Appl. Phys. Lett. 92, 133105 (2008).

${ }^{12}$ S. Murad and I. K. Puri, Appl. Phys. Lett. 95, 051907 (2009).

${ }^{13}$ S. Murad and I. K. Puri, Chem. Phys. Lett. 467, 110 (2008).

${ }^{14}$ A. I. Hochbaum, R. K. Chen, R. D. Delgado, W. J. Liang, E. C. Garnett, M. Najarian, A. Majumdar, and P. D. Yang, Nature 451, 163 (2008).

${ }^{15}$ J. Seyler and M. N. Wybourne, J. Phys.: Condens. Matter 2, 8853 (1990).

${ }^{16}$ L. L. Zhu and X. J. Zheng, EPL 88, 36003 (2009).

${ }^{17}$ E. Chilla, A. V. Osetrov, and R. Koch, Phys. Rev. B 63, 113308 (2001).

${ }^{18}$ W. Haiss, Rep. Prog. Phys. 64, 591 (2001).

${ }^{19}$ A. Rua, F. E. Fernandez, M. A. Hines, and N. Sepulveda, J. Appl. Phys. 107, 053528 (2010).

${ }^{20}$ X. Yi and H. L. Duan, J. Mech. Phys. Solids 57, 1254 (2009).

${ }^{21}$ J. E. Sader, J. Appl. Phys. 89, 2911 (2001).

${ }^{22}$ P. Sharma, S. Ganti, and N. Bhate, Appl. Phys. Lett. 82, 535 (2003).

${ }^{23}$ J. Lagowski, H. C. Gatos, and E. S. Sproles, Appl. Phys. Lett. 26, 493 (1975).

${ }^{24}$ G. F. Wang, and X. Q. Feng, Appl. Phys. Lett. 90, 231004 (2007).

${ }^{25}$ M. J. Lachut, and J. E. Sader, Phys. Rev. Lett. 99, 206102 (2007).

${ }^{26}$ K. P. G. M. Hu, J. V. Goicochea, X. Zhang, and D. Poulikakos, Nano Lett. 11, 618 (2011).

${ }^{27}$ Y. C. Wen, C. L. Hsieh, K. H. Lin, H. P. Chen, S. C. Chin, C. L. Hsiao, Y. T. Lin, C. S. Chang, Y. C. Chang, L. W. Tu, and C. K. Sun, Phys. Rev. Lett. 103, 264301 (2009).

${ }^{28}$ X. F. Wu, and Y. A. Dzenis, J. Appl. Phys. 100, 124318 (2006).

${ }^{29}$ F. Song, G. L. Huang, and V. K. Varadan, Acta Mech. 209, 129 (2010).

${ }^{30}$ A. I. A. I. Beltzer, Acoustics of Solids. (Springer-Verlag, New York, 1988).

${ }^{31}$ J. Zou, and A. Balandin, J. Appl. Phys. 89, 2932 (2001).

${ }^{32}$ H. I. Liu, D. K. Biegelsen, N. M. Johnson, F. A. Ponce, and R. F. W. Pease, J.Vac. Sci. Technol. B 11, 2532 (1993).

${ }^{33}$ T. Kizuka, Y. Takatani, K. Asaka, and R. Yoshizaki, Phys. Rev. B 72, 6 (2005).

${ }^{34}$ D. Lacroix, I. Traore, S. Fumeron, and G. Jeandel, Eur. Phys. J. B 67, 15 (2009).

${ }^{35}$ Y. Xiao, X. H. Yan, J. X. Cao, and J. W. Ding, J. Phys.: Condens. Matter 15, L341 (2003).

${ }^{36}$ D. Y. Li, Y. Y. Wu, P. Kim, L. Shi, P. D. Yang, and A. Majumdar, Appl. Phys. Lett. 83, 2934 (2003).

${ }^{37}$ M. J. Huang, W. Y. Chong, and T. M. Chang, J. Appl. Phys. 99, 043506 (2006).

${ }^{38}$ D. Donadio, and G. Galli, Nano Lett. 10, 847 (2010). 